\title{
Traduire
}

Revue française de la traduction

$220 \mid 2009$

Organisations internationales | Bicentenaire de Louis

Braille

\section{Traduction et organisations internationales : sortir de la bulle?}

Michel Rochard

\section{(2) OpenEdition}

Journals

Édition électronique

URL : http://journals.openedition.org/traduire/375

DOI : $10.4000 /$ traduire.375

ISSN : 2272-9992

Éditeur

Société française des traducteurs

Édition imprimée

Date de publication : 15 juin 2009

Pagination : 5-13

ISSN : 0395-773X

Référence électronique

Michel Rochard, «Traduction et organisations internationales : sortir de la bulle ? », Traduire [En ligne], 220 | 2009, mis en ligne le 12 novembre 2013, consulté le 19 avril 2019. URL : http://

journals.openedition.org/traduire/375; DOI : 10.4000/traduire.375 


\section{Traduction et organisations internationales : sortir de la bulle?}

\section{Michel Rochard}

Cet article fait suite à l'intervention présentée par l'auteur lors de la Journée de la traduction professionnelle 2008 à Lyon.

La situation des services de traduction, quelle que soit leur appellation (service, division, département, voire direction générale comme à la Commission européenne), est paradoxale.

Depuis quinze ans, les organisations internationales connaissent en effet de profondes restructurations assorties de réductions de coûts et de suppressions de postes. Les services de traduction n'y échappent pas. Depuis peu, le FMI est pris dans la tourmente et son service de traduction risque de connaître une cure d'amaigrissement drastique.

Pourtant, jamais les compétences exigées des traducteurs et réviseurs d'organisations internationales n'ont été aussi grandes. Que les organisations soient très spécialisées (Banque des règlements internationaux) ou au contraire pluridisciplinaires (comme la Commission européenne ou l'OCDE), l'actualité récente (crise des crédits hypothécaires à risque, suites du Protocole de Kyoto ou cycle de négociations de Doha) témoigne de la complexité des sujets traités par ces institutions dans un contexte de mondialisation.

Par ailleurs, dans les organisations ne comptant qu'une langue officielle (l'anglais) accompagnée de langues de travail (français, espagnol, etc.), les pages françaises ou autres des sites montrent bien le reflux de la traduction. Et ce n'est pas forcément mieux lorsqu'il y a plusieurs langues officielles.

Pourtant, la nécessité d'élaborer des solutions à des problèmes de portée mondiale devrait s'accompagner d'une augmentation des besoins de traduction professionnelle permettant de dépasser les contraintes d'une communication unilingue passant par le filtre d'un anglais international majoritairement pratiqué par des non-anglophones. Or, il n'en est rien.

Cette situation appelle plusieurs questions : pourquoi est-ce important d'avoir des traducteurs dans les organisations internationales ? L'analyse des coûts a-t-elle des conséquences irrémédiables? Que peuvent faire les traducteurs dans une telle situation? 


\section{Pourquoi avoir des traducteurs dans les organisations internationales?}

La question n'est pas anodine, car c'est un concentré de toutes les interrogations à l'égard du métier. Certes, on peut comprendre ceux qui répondront que cela n'a aucune importance, que c'est le problème d'une petite frange de traducteurs bien payés. On peut aussi considérer que ces organisations internationales sont devenues exclusivement anglophones et que l'existence d'autres langues officielles ou langues de travail n'est qu'une relique du passé et que leur maintien est économiquement injustifiable.

Ces deux raisonnements reflètent une part de réalité. Cela étant, on peut leur opposer d'autres arguments. Si la population de traducteurs d'organisations internationales est réduite et bénéficie de bonnes conditions de travail et de rémunération, c'est qu'elle constitue une sorte d'élite (on peut parfois compter une centaine de candidats par poste proposé à un concours). D'autre part, la présence de ces langues autres que l'anglais est une forme de garde-fou contre une uniformisation des esprits et des cultures. Ensuite, la maîtrise de l'anglais par le public non anglophone, si elle a progressé n'est sans doute pas aussi grande qu'on le pense et les experts non anglophones qui rédigent en anglais reconnaissent souvent qu'ils se restreignent dans leur expression par rapport à ce qu'ils écriraient dans leur langue maternelle. De plus, malgré tout, un minimum de traduction restera nécessaire, ce qui suppose le maintien d'une structure ou de procédures internes à cet effet. Enfin, sur le plan des coûts, aucun audit n'a réellement démontré que l'externalisation de services de traduction aboutissait à un meilleur rapport qualité-prix que le maintien des équipes existantes. Le cas de la Banque mondiale est emblématique, puisqu'on a procédé à une externalisation totale assortie d'une simple gestion administrative interne. Or, quelques années après, on a rétabli un service de traduction, certes moins étoffé, mais qui s'avérait indispensable.

Mais engager en ces termes le débat sur l'avenir des traducteurs des organisations internationales est réducteur.

D'abord, qu'on le veuille ou non, la traduction est un marché comportant plusieurs segments correspondant à plusieurs niveaux de rémunération et de coûts, ces deux notions étant déjà difficiles à apprécier en termes de comptabilité analytique et encore plus si l'on y ajoute les notions de qualité relative attendue (Prioux \& Rochard, 2007). C'est la logique de marché qui explique que le gonflement massif du nombre de diplômés de traduction fasse baisser les prix sur le segment déjà le moins rémunéré du marché (celui des traducteurs d'agence et/ou des traducteurs débutants). À l'inverse, il y a des segments sur lesquels l'entrée se fait par la sélection de compétences de haut niveau (les organisations internationales) avec des échelles de rémunération relativement stables en termes corrigés de l'inflation ou encore des segments très étroits où l'importance d'une communication de très grande qualité fait que le facteur prix devient presque secondaire par rapport au facteur qualité. Tout traducteur (sauf exception) aspire à être présent sur ces derniers segments du marché et on le comprend. 
Si l'on admet cette réalité, on peut se poser la question fondamentale de savoir comment les professionnels de la traduction, quel que soit le segment dans lequel ils travaillent, conçoivent l'avenir de leur métier à l'aube du $3^{e}$ millénaire, dans un contexte de mondialisation économique et culturelle, dans une économie où chaque activité doit justifier son existence en termes de coûts et de service.

Dans ces conditions, la traduction dans les organisations internationales relève d'une problématique que l'on retrouve dans tous les segments du marché et qu'aucun traducteur ne peut ignorer, d'autant que toutes les expériences de réduction radicale des ressources de traduction interne des organisations internationales ont uniquement profité aux agences de traduction qui font elles-mêmes pression sur les coûts de traduction des traducteurs indépendants. On peut donc poser la deuxième question.

\section{L'analyse des coûts est-elle synonyme de diminution des effectifs internes et d'externalisation intégrale?}

On peut effectivement se demander si les analyses de coûts effectuées à l'occasion d'audits déboucheront nécessairement sur une diminution radicale des effectifs des services de traduction des organisations internationales, voire sur une externalisation complète de la traduction dans ces organisations.

La situation actuelle incite en effet à le penser, puisque la position des services de traduction d'organisations internationales est régulièrement contestée par des audits essentiellement fondés sur des analyses de coûts. II faut en effet savoir que ces audits se multiplient et fragilisent toujours les services de traduction des organisations internationales. Si l'on met à part le cas de la Commission européenne pour laquelle l'élargissement de l'Union brouille un peu les cartes, on peut dire que la plupart des services de traduction des organisations internationales ont connu ou vont connaître des baisses d'effectifs.

Néanmoins, les services de traduction ont un certain nombre d'arguments à faire valoir. Dans ce genre de situation, il faut en effet réintroduire des considérations de coûts relatifs : la traduction externe est certes moins chère en termes bruts, mais si l'on prend en compte tous les coûts d'administration, de préparation des dossiers de traduction (ce que font rarement les entreprises commerciales pour leurs traducteurs externes) sans parler de révision, on constate que les coûts de la traduction externe et de la traduction interne se rapprochent sensiblement. Ensuite, les services de traduction ne font pas que de la traduction : selon les organisations, ils assurent des travaux terminologiques publiés, du "polissage " des textes originaux, des services de conseil linguistique aux autres directions, voire des services de documentation car ce sont souvent de véritables mémoires des organisations internationales. En outre, comme le dit Nicolas Froeliger dans son cours de méthodologie à l'Université de Paris 7, le traducteur 
intervient toujours à la pointe des savoirs, ce qui le rend " précieux " au sens propre comme au sens figuré. Par exemple, l'analyse de la crise des crédits hypothécaires aux emprunteurs à risque (les fameux subprimes) n'est compréhensible qu'à l'aide de connaissances affûtées des mécanismes de la titrisation, connaissances que possèderont des traducteurs internes suivant au jour le jour l'activité de l'organisation(1). Et quand une organisation internationale va publier un communiqué bilingue ou multilingue sur cette crise à l'issue d'une réunion, elle aura besoin d'une traduction techniquement incontestable et immédiatement disponible pour diffuser son message le plus largement possible. Tout recours à un intervenant extérieur présente alors un risque de sélection, de contrôle, de crédibilité et de disponibilité qui peut s'avérer largement plus onéreux que la mobilisation des traducteurs internes. Cette spécialisation a aussi des répercussions sur le plan quantitatif. En effet, le niveau de compétence exigé permet aussi aux traducteurs d'organisations internationales de parvenir à des chiffres de productivité tout à fait importants, à niveau de difficulté égal des textes. II y a donc là des arguments qui méritent d'être entendus y compris dans le cadre d'un audit. Bien entendu, l'idéal serait de pouvoir accorder une place centrale au facteur qualité de la traduction dans le cadre des audits. Mais il ne faut pas se leurrer, les " auditeurs " savent qu'ils n'auraient plus " la main " sur ce terrain et ils ne sont pas là pour parler qualité.

Bref, même si les traducteurs d'organisations internationales ne sont pas une espèce protégée, ils ne constituent pas nécessairement une espèce en voie de disparition. Ils ont un certain nombre d'atouts en main, mais ces atouts sont trop souvent occultés dans les situations de crise. Peut-être faut-il dans ces conditions s'interroger sur la propension des traducteurs d'organisations internationales, et plus généralement des traducteurs salariés, à vouloir se faire oublier selon l'adage "pour vivre heureux, vivons cachés " ? Effectivement, les traducteurs salariés ont parfois tendance à fonctionner dans une "bulle " éloignée des préoccupations de leur entreprise ou de leur organisation, mais aussi du reste de leur métier. Ces constats nous amènent à notre troisième question.

\section{Qu'y peuvent les traducteurs eux-mêmes ?}

Je pense que les traducteurs d'organisations internationales peuvent en fait beaucoup pour la promotion de leur métier et partant pour le maintien de leur propre situation. Et cette conviction vaut aussi pour tous ceux qui dans les métiers de la traduction se trouvent remis en cause ou risquent de l'être. Est-ce à dire qu'en tant que réviseur à l'OCDE, je serais à même d'offrir aux professionnels LA solution qui leur permettra de s'en sortir quoi qu'il arrive. Ce serait bien présomptueux.

(1) À l'OCDE, la crise des "subprimes " n'a pas été une surprise pour les traducteurs, car cela faisait deux ans au moins que nous avions traduit des documents sur les risques d'une telle crise dans le cadre d'un projet prémonitoire sur l'éducation financière des populations et une dizaine d'années que nous avions traduit des documents et une publication sur la titrisation. Le thème de la titrisation avait d'ailleurs fait l'objet, par notre intermédiaire, d'un exposé par un expert de l'OCDE lors d'un déjeuner de traducteurs financiers. 
Plus concrètement, les services de traduction de diverses structures publiques ou privées ont adopté un certain nombre de pratiques que je qualifierai d'exemplaires, en termes d'autoformation, de veille terminologique, de recherche documentaire, etc. Nous pourrions tous nous en inspirer quel que soit le segment du marché dans lequel nous opérons, à commencer par le jeune diplômé qui travaille au début pour des agences, mais qui entend bien s'en sortir.

Ensuite, s'ils ne veulent pas se condamner eux-mêmes à subir passivement les audits, les directives, les lois et décrets divers, les traducteurs d'organisations internationales doivent être actifs et réactifs. Pour ce faire, ils doivent commencer par sortir de leur " bulle ", par rompre avec leur traditionnelle " invisibilité "(2).

Trois axes peuvent permettre de sortir de la bulle : assumer des fonctions de cadre au sens des entreprises, développer les relations avec la profession, enfin mieux s'intégrer dans leur propre organisation.

\section{Être traducteur, c'est se comporter en cadre}

II faut d'abord que les traducteurs mettent en adéquation leurs ambitions et leurs comportements. Tous les traducteurs salariés veulent obtenir, on les comprend, le statut de cadre, ce qui est en France synonyme d'un certain nombre d'avantages et ce qui est un facteur de reconnaissance. Mais avoir un statut est une chose, assumer des fonctions de cadre en est une autre et cela semble indispensable. II arrive certes que des traducteurs assument concrètement ces fonctions, mais dans bien d'autres cas, être cadre signifie simplement bien faire son travail. Cette attitude est encore plus problématique dans le cas d'organisations internationales où les réviseurs se conçoivent souvent plus comme des "super-traducteurs "à la compétence professionnelle difficilement contestable, que comme des " cadres " au sens où on l'entend dans une entreprise.

II faut rompre avec la logique du " super-traducteur " anonyme. La révision est bien entendu le vecteur privilégié pour y parvenir : révision interne des traducteurs juniors, relecture entre pairs sur un projet commun, révision des traducteurs externes. Ensuite, ces différentes formes de révision doivent donner lieu à un retour d'information à destination du révisé. Chaque fois que c'est possible, le retour d'information doit être assuré directement par le réviseur. C'est bien entendu évident dans le cadre de la révision interne, mais c'est aussi possible dans le cadre de la révision de traducteurs externes, notamment ceux qui travaillent fréquemment dans le domaine spécialisé du réviseur.

(2) Le modèle de l'invisibilité est sans doute le personnage du traducteur dans l'Argent de Zola, qui ne voit personne, qui est voué à être mal payé et qui va mourir phtisique tout seul dans sa chambre de bonne glaciale sous les toits. C'est l'antithèse du traducteur moderne, celui qui pratique l'un des métiers qui s'est le plus rapidement adapté à l'Internet et aux techniques avancées de l'information et de la communication. 
Toujours en ce qui concerne les suites de la révision, il est bon que les réviseurs formulent une évaluation du travail des révisés, ce que les traducteurs sont souvent réticents à faire. Cela aussi fait partie de leur mission de cadre.

Grâce à la connaissance des traducteurs externes ou internes par la révision, au retour d'information et à l'évaluation, le réviseur (3) se mue en un animateur d'équipe, celui qui devient la référence pour tout un ensemble de traducteurs, celui vers lequel les responsables de la répartition du travail vont se tourner lorsqu'il s'agit de préparer une grande réunion, de donner de la cohérence à un gros projet ou une étude phare.

En développant toutes les formes de révision, on peut créer des équipes, introduire une culture de l'encadrement pédagogique, préparer les traducteurs internes à sortir de la bulle.

\section{Développer les relations avec la profession}

Autre manifestation de l'existence d'une bulle, on observe une certaine étanchéité entre le monde des traducteurs indépendants, d'une part, et celui des traducteurs salariés en général et des traducteurs d'organisations internationales, de l'autre. Cette absence de relations est préjudiciable à tous les secteurs de la profession. En effet, on l'a dit, il ne faut pas se leurrer, les réductions brutales des effectifs des organisations internationales aboutissent rarement à un gonflement de la demande de traduction pour les traducteurs indépendants et à une augmentation des prix. L'externalisation massive s'exerce généralement au profit des agences de traduction ayant la plus grosse force de frappe économique, ce qui n'est guère réjouissant pour la profession et la qualité des traductions.

Je pense donc qu'il faut encourager les traducteurs d'organisations internationales à s'ouvrir vers la profession. II ne s'agit ni d'institutionnaliser, ni de systématiser cette ouverture, mais d'instaurer progressivement une culture interne au service qui intègre cette dimension. Le séminaire organisé par le service de traduction de l'OCDE à l'intention de ses traducteurs externes avec la participation de représentants d'associations professionnelles et de formations de traduction est une bonne illustration de cette démarche.

Bien entendu la révision est une fois encore un lien entre les traducteurs d'organisations internationales et la profession. Ce lien découle du recours à la traduction externe. Lorsque ses compétences ont été établies par les réviseurs, le traducteur externe doit être considéré comme membre de l'équipe à part entière et non comme un bouche-trou en cas de demande excédentaire. Le retour d'information et la communication d'éléments documentaires aux traducteurs externes sont l'une des clés de cette intégration. C'est également dans ce souci d'intégration que les réviseurs doivent dans toute la mesure du possible non seulement

(3) Lorsque je parle ici de "réviseur ", je ne songe pas à un grade dans une hiérarchie, mais à une fonction qui doit bien entendu être assumée par des réviseurs en titre, mais qui peut l'être aussi par des traducteurs confirmés vis-à-vis de traducteurs externes ou de traducteurs juniors. 
entretenir le contact avec les traducteurs externes (par des révisions annotées) mais encore " animer " l'équipe externe comme ils doivent le faire avec l'équipe interne. C'est le réviseur qui doit apporter le " plus " que ne donnent pas les sites extranet destinés aux traducteurs externes. Le " plus " ce sont les éléments de contexte, le document le plus important et/ou le mieux traduit du dossier documentaire, la logique du programme de travail spécifique dont fait partie le document à traduire, les concepts-clés de ce programme.

La relation avec les traducteurs externes doit être aussi fondée sur le respect. Dans un souci pédagogique, on peut ainsi faire réviser (relire) de bons traducteurs externes par des traducteurs internes " junior ". Cela peut être un exercice stimulant permettant d'aiguiser la vigilance des jeunes traducteurs internes tout en leur apprenant à apprécier la qualité du travail de professionnels ne disposant pas de tous les moyens qu'apporte le fait d'être au sein d'une organisation très structurée.

Les autres démarches en direction de la profession peuvent être des plus variées : participation à diverses manifestations (par exemple les fameux " déjeuners de traducteurs financiers "), participation à des listes de discussion et autres forums de traduction et de terminologie. Dans ce dernier cas, il peut y avoir des échanges fructueux, car tous les traducteurs spécialisés assurent naturellement une "veille " de leur domaine et le partage de connaissances entre traducteurs indépendants et salariés ou traducteurs d'organisations internationales peut être alors particulièrement utile (Rochard, 2006).

II y a aussi la participation de traducteurs d'organisations internationales à des colloques, séminaires ou congrès : en tant qu'intervenants pour les plus aguerris, en tant que simples auditeurs pour les traducteurs " juniors ". C'est un moyen de dire à la profession que nous en faisons partie, que nous respectons ses initiatives et que nous nous y associons.

Il y a enfin l'enseignement qui est une démarche extrêmement enrichissante entre un traducteur et des apprentis. C'est aussi une école de formation à la révision pédagogique et à l'encadrement.

Naturellement, cette ouverture sur la profession ne doit pas être anarchique et risquer de mettre en péril les équilibres propres à un service de traduction. Néanmoins, il s'agit là de considérations qui doivent être analysées en termes d'utilité de l'échange pour la visibilité du service, la formation des équipes internes et externes de traducteurs, la diffusion de bonnes pratiques, le partage de connaissances, etc. C'est d'ailleurs la condition pour que la participation de traducteurs d'organisations internationales à de tels échanges soit fructueuse pour la profession.

\section{S'intégrer dans l'organisation}

Ce troisième axe pourrait aussi s'intituler "l'ouverture sur l'intérieur ". Les traducteurs sont largement inconnus des autres services des organisations internationales et ils apparaissent trop souvent comme des gens "à part ". Casser cette image paraît indispensable pour plusieurs raisons. 
D'abord, parce que vouloir vivre " cachés " au sein de l'organisation, c'est s'exposer comme des candidats naturels à des mesures de rationalisation.

Les traducteurs d'organisations internationales ont aussi intérêt à travailler directement avec les experts pour mieux gérer les flux d'information sur les textes à traduire en intervenant en amont, au moment où un programme de travail se dessine, pour en connaître et en comprendre les enjeux. Ce travail avec les experts est aussi un moyen de leur faire voir que les traducteurs sont des gens qui " pensent " et non des machines à traduire. C'est d'ailleurs ce qu'ils font souvent en indiquant aux experts des erreurs dans leurs textes, et les experts les en remercient généralement. Les services de traduction peuvent faire beaucoup mieux en incitant les réviseurs à se présenter physiquement aux équipes d'experts, à aller les voir, à définir des procédures de travail communes. II est aussi possible de détacher de temps en temps pour quelques semaines ou quelques mois des traducteurs spécialisés afin de travailler en prise directe avec les experts.

Lorsque ce type de relations s'instaure, on constate que les experts contactent spontanément les traducteurs pour leur faire comprendre l'importance d'un texte, discuter de la terminologie, voire négocier des délais raisonnables.

Enfin et c'est un vecteur très important de relations entre traducteurs et experts, toutes les organisations internationales publient des travaux des plus divers touchant peu ou prou à la terminologie. La nécessité de se comprendre dans le cadre de la mondialisation impose en effet aux experts de définir les concepts qu'ils utiliseront dans leur dialogue entre eux et cette discussion amène nécessairement une démarche multilingue. Les traducteurs d'organisations internationales ne doivent pas attendre que les experts décident unilatéralement de la terminologie à utiliser. lls doivent être des acteurs de la production terminologique, le cas échéant en synergie avec les terminologues. Les traducteurs font leur propre veille terminologique et comme ils sont les premiers à utiliser concrètement les termes nouveaux dans leurs textes, ils savent mieux que quiconque si un choix terminologique est viable ou non. Ils doivent s'intégrer dans la réflexion terminologique le plus en amont possible et apporter à leur organisation leur compétence dans ce domaine.

\section{Pour conclure}

La grande leçon que l'on peut tirer des expériences négatives subies par les traducteurs d'organisations internationales et les traducteurs en général, c'est qu'ils ne doivent pas se lamenter sur le bon vieux temps disparu, ni attendre que les choses leur tombent sur la tête de façon fataliste, qu'il s'agisse des textes ou des décisions managériales. II s'agit au contraire d'affronter l'avenir en valorisant la profession. Les traducteurs d'organisations internationales, les traducteurs salariés et les indépendants doivent pour ce faire travailler résolument à améliorer 
leur visibilité, à donner une meilleure image d'eux-mêmes. Ils doivent s'inscrire dans une démarche dynamique et exemplaire. Dans cette démarche, ils doivent envisager la traduction certes comme un marché à plusieurs segments pour ne pas se tromper de cible, mais aussi comme un métier, c'est-à-dire une chaîne d'intérêts communs.

Michel Rochard est titulaire d'un diplôme de traduction de l'Université de Mayence à Germersheim et d'un doctorat de traductologie de l'université Paris 3 (ESIT). Réviseur à la Division de la traduction de l'Organisation de coopération et de développement économiques (OCDE), il est aussi chargé de cours de traduction économique et financière à l'Université de Paris 7 (EILA).

\section{Bibliographie :}

PRIOUX René et ROCHARD Michel (2007), "Économie de la révision dans une organisation internationale : le cas de l'OCDE ", in : Brunette et al. Interviews with translation professionals, Journal of Specialised Translation, $\mathrm{n}^{\circ}$ 8, juin 2007, http://www.jostrans.org/about.php

ROCHARD Michel (2006), "La rétroconception entre compréhension et ré-expression des textes économiques ", in : Élisabeth Lavault-Olléon (éd.), Traduction spécialisée : pratiques, théories, formations, Travaux interdisciplinaires et plurilingues en langues étrangères appliquées, vol. 10, Peter Lang, Berne, 2006. 